\title{
Lateral interactions in visual perception of temporal signals: cortical and subcortical components
}

\author{
Claudio E. C. Teixeira ${ }^{1,2}$, Luiz Carlos L. Silveira ${ }^{1,2}$ and Jan Kremers ${ }^{2,3}$ \\ 1 - Universidade Federal do Pará, Belém, PA, Brazil \\ 2 - University of Erlangen - Nürnberg, Erlangen, BY, Germany \\ 3 - University of Bradford, Bradford, WYK, UK
}

\begin{abstract}
The aim of this work was to isolate and investigate subcortical and cortical lateral interactions involved in flicker perception. We quantified the perceived flicker strength (PFS) in the center of a test stimulus which was simultaneously modulated with a surround stimulus (50\% Michelson contrast in both stimuli). Subjects were requested to adjust the modulation depth of a separate matching stimulus that was physically identical to the center of the test stimulus but without the surround. Using LCD goggles, synchronized to the frame rate of a CRT screen, the center and surround could be presented monoptically or dichoptically. In the monoptic condition, center-surround interactions can have both subcortical and cortical origins. In the dichoptic condition, center-surround interactions cannot occur in the retina and the LGN, therefore isolating a cortical mechanism. Results revealed both a strong monoptic (subcortical plus cortical) lateral interaction and a weaker dichoptic (cortical) lateral interaction. Subtraction of the dichoptic from the monoptic data revealed a subcortical mechanism of the lateral interaction. While the modulation of the cortical PFS component showed a low-pass temporal-frequency tuning, the modulation of the subcortical PFS component was maximal at $6 \mathrm{~Hz}$. These findings are consistent with two separate temporal channels influencing the monoptic PFS, each with distinct lateral interactions strength and frequency tuning characteristics. We conclude that both subcortical and cortical lateral interactions modulate flicker perception. Keywords: temporal processing, monoptic lateral interactions, dichoptic lateral interactions, cortical interactions, subcortical interactions, flicker perception.
\end{abstract}

Received 21 December 2011; received in revised form 4 March 2011; accepted 5 March 2011. Available on line 15 June 2011

\section{Introduction}

In the primate lateral geniculate nucleus (LGN), the response amplitudes of $\mathrm{M}$ and $\mathrm{P}$ cells are influenced by the interactions between their receptive field (RF) centers and surrounds. With the center and surround stimuli matching the cells' classical RFs' centers and surrounds, the strength of these interactions depend on the relative modulation phase between the two stimuli (Kilavik, Silveira, \& Kremers, 2003; Kremers, Kozyrev, Silveira,

Claudio E. C. Teixeira, Biological Sciences Institute, Tropical Medicine Nucleus, Federal University of Pará, Belém, Pará, Brazil, Dept. of Ophthalmology, University Hospital Erlangen, University of Erlangen-Nürnberg, Germany. Luiz Carlos L. Silveira, Biological Sciences Institute, Tropical Medicine Nucleus, Federal University of Pará, Belém, Pará, Brazil. Jan Kremers, Dept. of Ophthalmology, University Hospital Erlangen, University of Erlangen-Nürnberg, Germany, School of Life Sciences, University of Bradford, Bradford, UK. Correspondence regarding this article should be directed to: Dr. Jan Kremers, Dept of Ophthalmology, University of Erlangen-Nürnberg, Schwabachanlage 6, 91054 Erlangen, Germany. E-mail: jan.kremers@uk-erlangen.de
\& Kilavik, 2004; Kozyrev, Silveira, \& Kremers, 2007). Psychophysically, the perceived flicker strength (PFS) in a central field simultaneously modulated with a surround depends not only on the physical modulation contrasts in the central and surrounding fields, but also on their relative modulation phase. The PFS is strong when center and surround stimuli modulate in counter-phase and weak when the two modulate in-phase (Kremers et al., 2004; Kozyrev et al., 2007; Kremers \& Rimmele, 2007).

However, the similarities between cell responses and psychophysical PFS in a central stimulus go beyond these superficial analogies. First, owing to small differences in the response latencies of LGN RFs center and surround, the antagonism between the two changes with temporal frequency. Temporal frequency has a similar influence upon the PFS in the central stimulus (Kremers et al., 2004). More recently, a model linking the responses of an array of LGN cells with different RFs locations to perception was described (Kozyrev et al., 2007), what led to the proposal that the physiological basis of PFS can be found already in subcortical structures.

Although the similarities between subcortical physiology and perception are compelling, a more definite 
proof that the two are related is lacking. Recently, additional evidence of the importance of subcortical structures was obtained by comparing the interactions between center and surround stimuli under two conditions (D'Antona, Kremers, \& Shevell, 2008). In the first condition, the two stimuli were presented monoptically. In this case, the interaction can take place at the subcortical and the cortical levels (Figure 1A). In the second condition, the two stimuli are presented dichoptically and the interaction can only have a cortical origin since the signals from the two eyes merge at a cortical level (Figure 1B). The difference between monoptic and dichoptic data extracts the subcortical component in lateral interactions. In both conditions, subjects matched the PFS in the center test stimulus by adjusting the modulation depth in a matching stimulus that was physically identical to this center stimulus but without the surround. It was found that the PFS depends more strongly upon the relative phase between center and surround stimuli when the two are presented monoptically than when presented dichoptically (D'Antona et al., 2008). The interpretation of the data by these authors is that cortical interactions are stronger than subcortical interactions and play a major role in the monoptic PFS (D'Antona, Kremers, \& Shevell, 2011 - in submission).

However, the psychophysical approach used by D'Antona et al. (2011) possibly influenced their data. In their work, the PFS was measured by using a test stimulus of which the center and surround were always presented to one eye (either to the same eye in monoptic or to different eyes in dichoptic conditions) but the matching stimulus was presented to the two eyes. Owing to inter-ocular interaction, the contrast in the matching field might be influenced by this type of presentation. As a result, the dynamic range of matching stimuli decreases and might make the results of PFS measurements less accurate. In the present study, we decided to quantify the PFS measurements by using a monocularly presented matching field (Figure 1).

Our results confirm the data of D'antona et al. (2011), i.e. the PFS depends more strongly upon the relative phase between center and surround stimuli when the two are presented monoptically, indicating the presence of cortical and subcortical mechanisms of lateral interactions. However, we find larger subcortical components than they do.

Parts of the results were previously presented in an abstract form (Teixeira \& Kremers, 2009).

\section{Methods}

\section{Participants}

Three healthy subjects participated as observers in the present study, two of the authors (CT and JK,
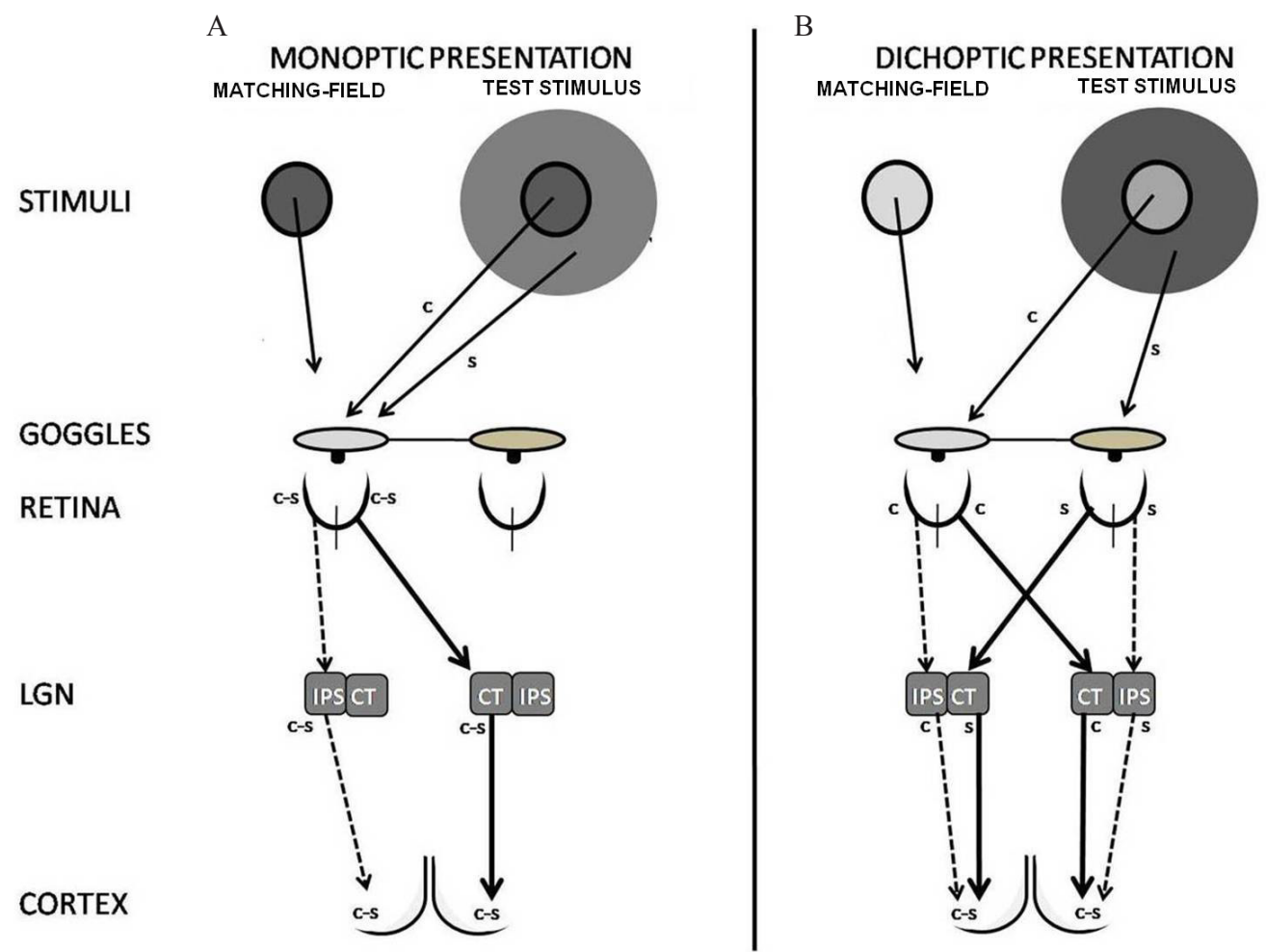

Figure 1. Test stimulus and matching-field presentations in monoptic $(\mathrm{A})$ and dichoptic $(\mathrm{B})$ conditions and and the putative respective sites of possible lateral interactions in the visual pathway. c, center; s, surround; c-s, center-surround interactions; IPS, ipsilateral; CT, contralateral. The center and surround test stimuli have $1^{\circ}$ and $3^{\circ}$ outer diameter, respectively. The matching field has $1^{\circ}$ outer diameter. 
ages 32 and 50, respectively) plus one naïve subject (GP, age 32). All subjects had normal color vision and underwent an extensive ophthalmological examination. There were no signs of retinal disorders. All the psychophysical measurements were performed under dark-adapted conditions using natural pupils and full optical correction. All experimental procedures adhered to the Helsinki Declaration.

\section{Apparatus}

The stimuli were presented on an EIZO L360 monitor controlled by a Matrox Millenium G550 graphic card using commercially available software (VisionWorks $^{\mathrm{TM}} 4.0$ for Windows). Two different stimuli (a test stimulus and a matching stimulus) were displayed simultaneously (Figure 1).

The test stimulus consisted of a spatially homogenous circular center and a spatially homogenous annular surround. The outer diameters of the center stimulus and of the surround were $1^{\circ}$ and $3^{\circ}$, respectively. There was a small annular gap $\left(0.1^{\circ}\right)$ between the center and the surround. This annular gap was included to enable a perceptual separation of the center circle from its surround at all conditions. In addition, to reduce residual flicker in the center circle produced by highfrequency temporal modulation at the edge between center and surround stimuli (Kremers et al., 2004). The distance between the outer diameters of the surround test stimulus and the matching stimulus was $5^{\circ}$.

While the center and surround stimuli had equal mean luminances $(45 \mathrm{~cd} / \mathrm{m})$ and chromaticities $(10,30$, and $5 \mathrm{~cd} / \mathrm{m}$ mean luminance of the red, green, and blue phosphors, respectively, resulting in a white with CIE 1964 coordinates: $\mathrm{X}=0.3330, \mathrm{Y}=0.3263$ ), the background had slightly higher mean luminance $(50 \mathrm{~cd} / \mathrm{m})$ but with the same chromaticity $(11.11,33.33$, and $5.55 \mathrm{~cd} / \mathrm{m}$ mean luminance of the red, green, and blue phosphors, respectively, resulting in a white; CIE 1964 coordinates were $\mathrm{X}=0.3330, \mathrm{Y}=0.3263$ ). Stray light would result in an increased sensitive to in-phase modulation of center and surround stimuli and a decreased sensitivity to counterphase modulation. However, the results are reversed strongly suggesting that stray light has a minor effect on the mechanisms under investigation in this work (see below).

The stimuli were viewed through goggles with LCD shutters (NuVision 60GX, NuVision Technologies Inc.). An infrared emitter synchronized the LCD shutters to the monitor's refresh rate. When the shutters were opened, they transmitted $20 \%$ of the light. As a result, we can estimate that the center and surround stimuli had mean luminance of $9 \mathrm{~cd} / \mathrm{m} 2(2,6$, and $1 \mathrm{~cd} / \mathrm{m} 2$ mean luminance of the red, green, and blue phosphors, respectively; the shutters did not alter the stimulus' chromaticity because the LCD shutters were spectrally neutral).

The luminance of the center and surround stimuli was sinusoidally modulated in time with $50 \%$ Michelson contrast. This relatively high contrast was chosen to get reliable psychophysical data at all stimulus conditions. As the contrast of the test stimulus was constant at all conditions, contrast dependent saturation does not influence the results. The measurements were performed at three temporal frequencies $(3,6$, and $12 \mathrm{~Hz})$. The center and surround stimuli had identical temporal frequencies, time averaged luminances, and time averaged chromaticities.

The matching stimulus consisted of single stimulus with the same shape, size, temporal frequency, time averaged luminance, and time averaged chromaticity as the central circle of the test stimulus. The contrast of the matching stimulus was variable and set by the observer until the PFSs in the matching stimulus and in the center of the test stimulus were matched.

The matching stimulus and the center of the test stimulus were presented to the left eye. The measurements were performed in two conditions: one in which the surround of the test stimulus was presented also to the left eye (monoptic condition) and one in which it was presented to the right eye (dichoptic condition) (Figure 1). While the surround stimulus was presented selectively to the left or right eye, an unmodulated light of equal time averaged luminance was presented to the other eye.

\section{Procedure}

The PFS measurements were done randomly at 13 relative center-surround test stimulus phases: $-180^{\circ}$, $-150^{\circ},-120^{\circ},-90^{\circ},-60^{\circ},-30^{\circ}, 0^{\circ}, 30^{\circ}, 60^{\circ}, 90^{\circ}, 120^{\circ}$, $150^{\circ}$ and $180^{\circ}$ (Figure 2). A two-alternative forcedchoice method was used to match the PFS in the matching stimulus to the one in the center of the test stimulus in both the monoptic and dichoptic presentation conditions. Subjects were requested to indicate, by pressing a button, whether the perceived flicker in the test stimulus was stronger or weaker than the perceived flicker in the center of the matching stimulus. In each PFS measurement run, subjects viewed the matching stimulus and the center test stimulus, free to make eye and small head movements, for as long as necessary to make a perceived-flicker judgment.

In each PFS measurement, the contrast in the matching stimulus was varied and the test stimulus was not altered. Each time the subject indicated that the perceived flicker in the matching stimulus was stronger than in the center test stimulus, the matching stimulus contrast was decreased. It was increased each time subject indicated that the perceived flicker of the matching stimulus was weaker than in the center test stimulus.

Two staircases, one starting at $0 \%$ and the other at $100 \%$ contrast, were used. The contrasts in the matching stimulus were initially changed in $10 \%$ steps. After a first change in response (from a weaker to a stronger PFS in the test stimulus or vice versa), the direction of contrast change was reversed and the step size was decreased 


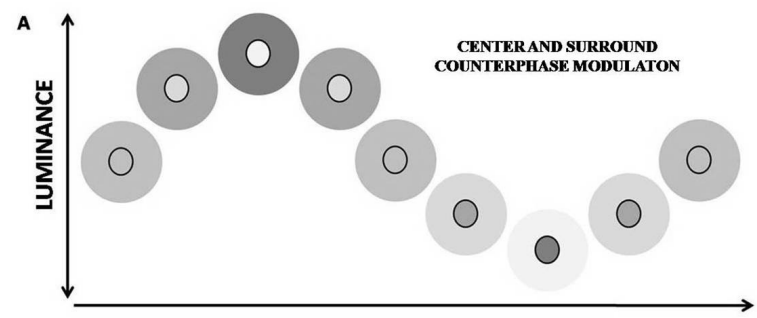

TIME

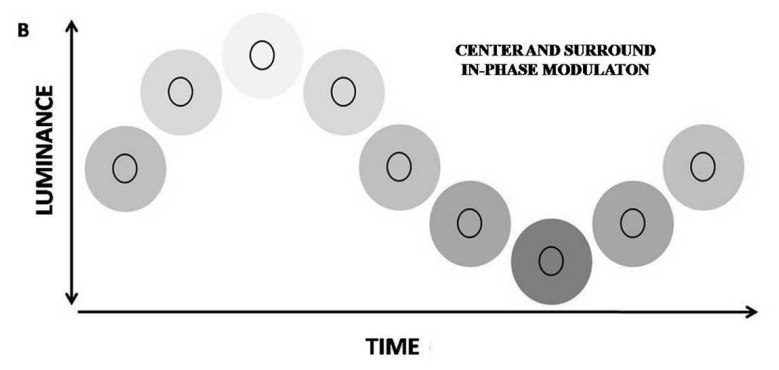

Figure 2. Schematic diagram showing one modulation cycle of the center and surround stimuli for counterphase (A) and in-phase (B) modulation.

to $5 \%$. Subsequent changes in response led to a change in step direction and further decreasing step sizes $(3 \%$, $2 \%$, and $1 \%$ respectively). Once a $1 \%$ contrast step was reached two additional changes in response resulted only in a direction reversal without a change in step size. After six changes in response, it was assumed that the PFSs in the matching stimulus and in the center test stimulus matched for that staircase. Thus, in each PFS measurement run two independent estimates of the PFS in the center test stimulus were obtained (one from each staircase). A PFS measurement run was always completed within one session. Each run was repeated three times. The means and standard deviations of the six matching PFS estimates from these three runs using two staircases were calculated. The standard deviation of the results shows that PFS's could be estimated with $10 \%$ reliability. However, the reliability was much better in most cases.

\section{Results}

\section{Perceived flicker strength}

Figures 3-5 (top panels) display the mean PFS as a function of the phase difference between center and surround for the three subjects, at three different temporal frequencies $(3,6$ and $12 \mathrm{~Hz})$ for the monoptic and dichoptic conditions. The PFS depends strongly on relative center-surround phase. There was a range of relative phase differences (between $-30^{\circ}$ and $30^{\circ}$ ) in which almost no flicker was perceived in the center stimulus in the monoptic test condition. In the dichoptic condition, flicker was perceived at all phase differences. The modulation of the PFS (i.e. the change of PFS as a function of relative phase) quantifies the strength of the lateral interaction. The modulation of the PFS was larger in the monoptic stimulus presentations at all temporal frequencies. The modulation of the dichoptic PFS decreases between 3 and $12 \mathrm{~Hz}$.

As mentioned above, the PFS modulation in the dichoptic condition probably has a cortical origin. The PFS modulation in the monoptic condition can have cortical and subcortical origins. Thus, for each subject, we estimated the subcortical component contribution to the phase dependent PFS by subtracting the dichoptic PFS from the monoptic PFS data. We implicitly assume that the two components do not interact and are completely independent. The estimated subcortical PFSs are shown in the lower panels of Figures. 3-5. The modulation of the dichoptic (cortical) PFS component is similar or smaller than the modulation of the estimated subcortical PFS component at $6 \mathrm{~Hz}$.

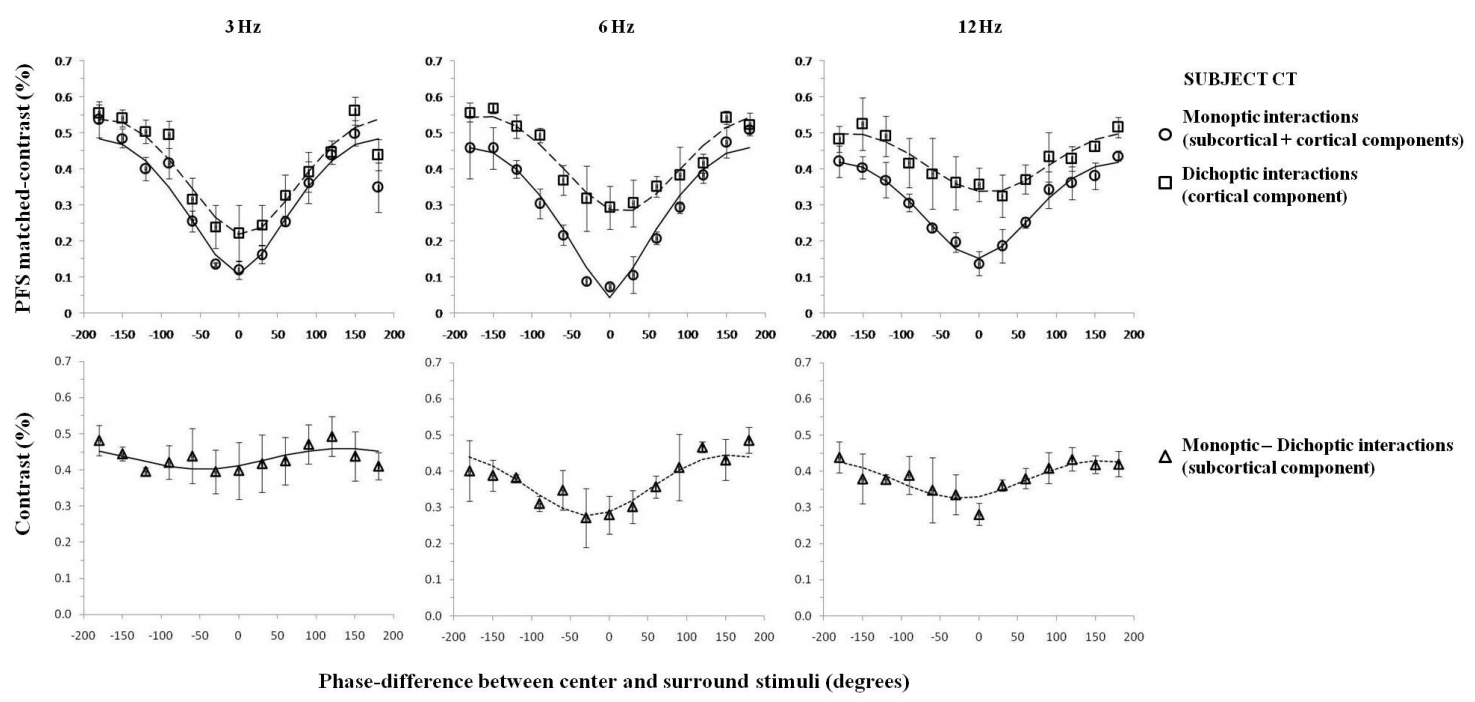

Figure 3. Top: The PFS (mean of six measurements \pm SD) in the center test stimulus plotted as a function of the phase difference between center and surround stimuli at all temporal frequencies for subject CT. Bottom: Subtracting the dichoptic response from the monoptic response estimates the subcortical PFS component. The curves are fits of Equation (1) to the data. 


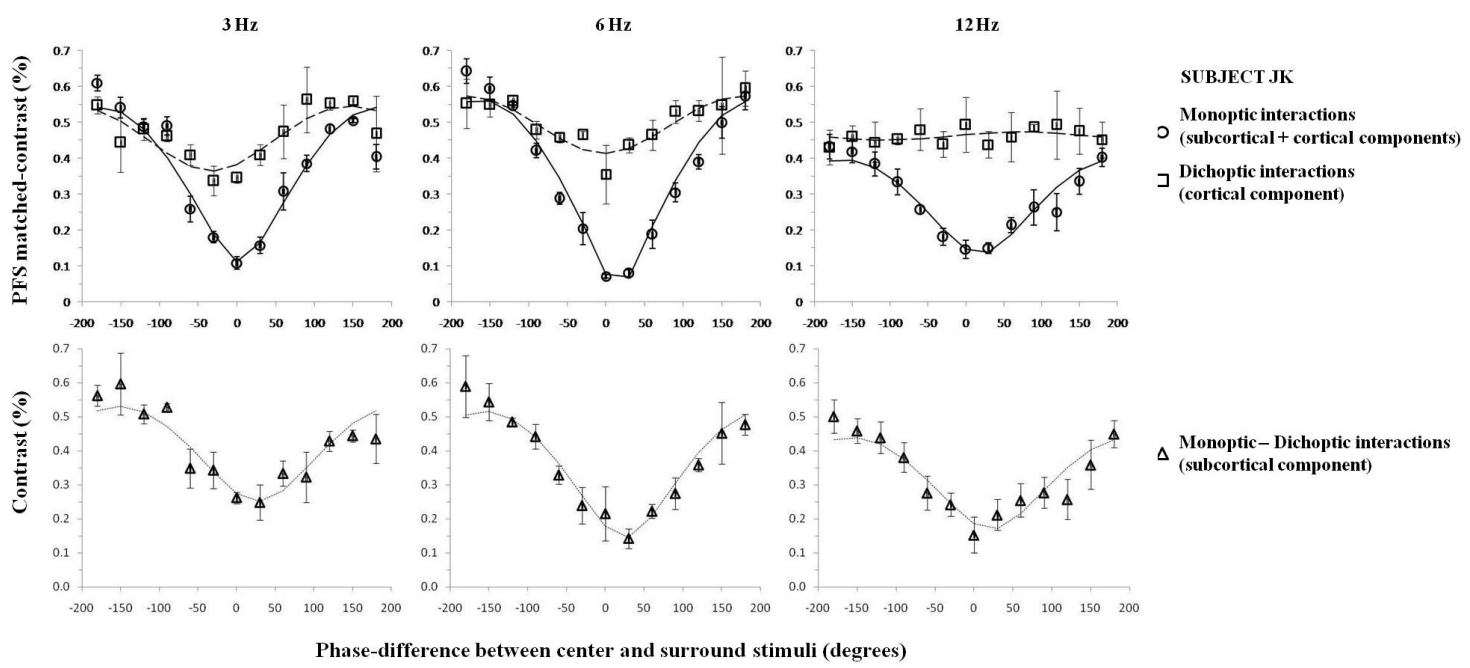

Figure 4. The same data as in Figure 3 for subject JK.

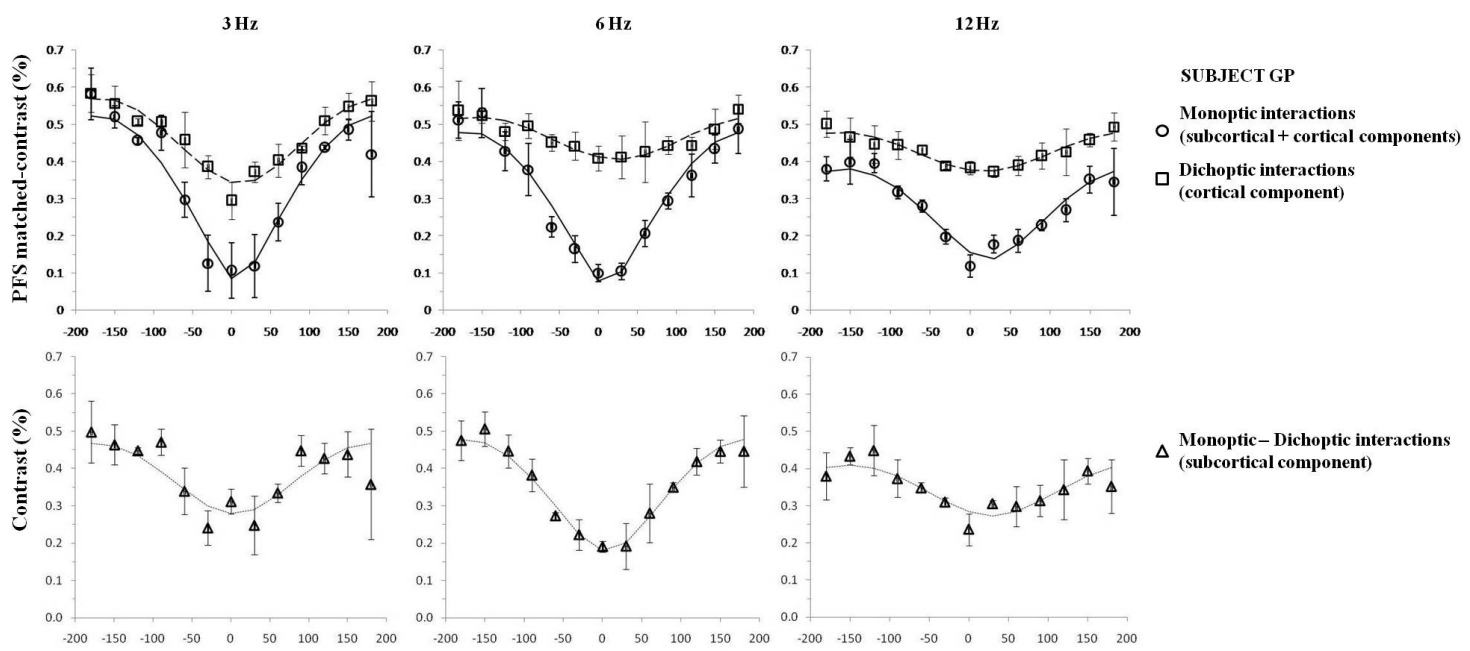

Phase-difference between center and surround stimuli (degrees)

Figure 5. The same data as in Figure 4 for subject GP.

\section{Linear vector addition model}

In prior work, it was found that the responses of LGN neurons are adequately modeled by a linear vector addition of the responses to the center and to the surround stimuli. Assuming that the linear addition is also applicable to describe the perceived flicker strength, the psychophysical data were fitted by Equation 1 (Kremers et al., 2004; Kozyrev et al., 2007):

$$
P F S=\sqrt{R_{C}^{2}+R_{S}^{2}-2 R_{C} \times R_{S} \times \cos (S-P)}
$$

,where $R_{C}$ and $R_{S}$ are the selective psychophysical responses to the center and surround stimuli, respectively. As the amplitude modulation of the PFS in the center test field depends on the response to the surround field, which in turn depends on its spatial extent (Kremers \& Rimmele, 2007; Teixeira \& Kremers, 2009), $R_{S}$ quantifies the PFS modulation and thus the strength of lateral interactions. $S$ is the phase of the surround stimulus relative to the phase of the center stimulus, and $P$ is the relative phase of the response to center and surround stimuli at a minimal PFS. The curves in Figures 3-5 are fits of this model to the data. Equation 1 was fitted to the data using the Solver routine of the Microsoft $C$ Excel 2007 program. The fitting routine changed the free parameters $R_{C}, R_{S}$ (both expressed in Michelson contrast) and $P$ (expressed in degrees) to minimize the sum of squared distance between model fit and experimental data at each relative stimulus phase. From the fits of the model, we obtained estimates of the three free parameters, $R_{C}, R_{S}$ and $P$. Here, these parameters do not symbolize the psychophysical instead of physiological responses. Thus, as we describe the perception data in terms of equivalent contrasts, $R_{C}$ and $R_{c}$ are expressed not in spikes per second but in Michelson contrast.

The psychophysical data were described satisfactorily by this model. However, the phases were not well constrained when the modulation of the PFS (quantified by $R_{S}$ ) was 
small. Therefore, we disregarded the phase estimates from those fits in which the difference between the maximal and minimal values of the PFS equivalent contrast was less than 3 times the average of the standard deviations at all data points. All phase estimates from the fits to the data could be used, except for JK's dichoptic PFS at $12 \mathrm{~Hz}$ and for CT's subcortical PFS at 3Hz (Figure 6). Table 1 gives all estimates obtained from the fits of Equation 1.
Figure 6 (top panels) displays the estimates of free parameters as a function of temporal frequency. The upper panels show the estimated $R_{C}$ values. These were always larger in the dichoptic than in the monoptic stimulus conditions as a function of temporal frequency. The $R_{C}$ values for the subcortical mechanisms do not bear information because they are influenced by the subtraction of the two sets of experimental data. Therefore they are

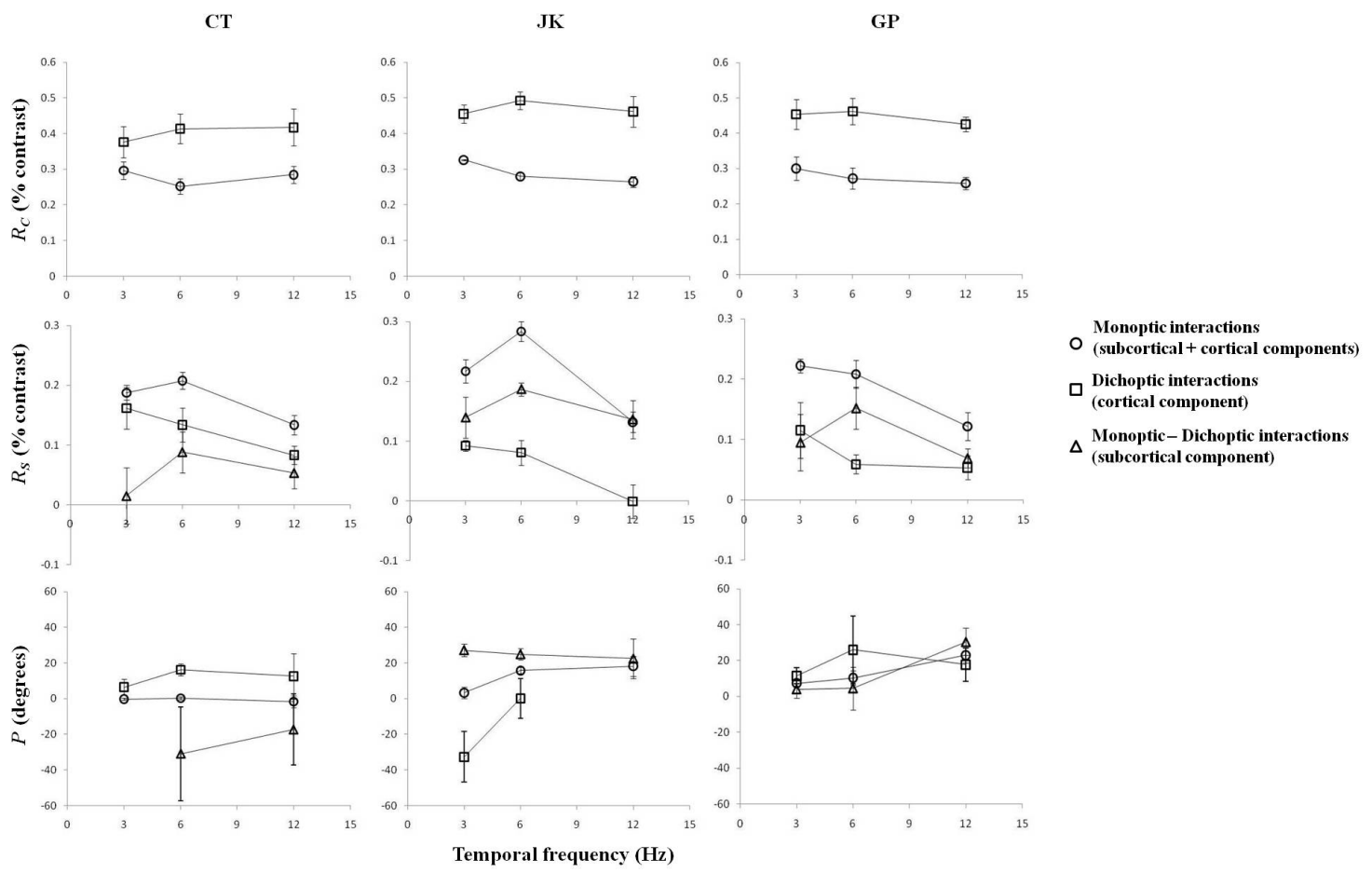

Figure 6. Estimates of $R_{C}$ (top), $R_{S}$ (middle), and $P$ (bottom) as a function of temporal frequency for three subjects at all test conditions (see also Table 1). Note that estimates from the fits related to the dichoptic PFS at $12 \mathrm{~Hz}$ for subject JK and related to the estimated subcortical PFS component at $3 \mathrm{~Hz}$ for subject CT were disregarded (see Linear Vector Model section).

Table 1. Averaged parameters $R_{C}, R_{S}$ (in \% contrast) and $P$ (in degrees) estimated from the fits of Equation 1 to the psychophysical data at three temporal frequencies and for three subjects.

\begin{tabular}{|c|c|c|c|c|c|c|c|c|c|c|c|}
\hline Monoptic $R_{C}$ & $\mathbf{C T}$ & JK & GP & Monoptic $\boldsymbol{R}_{S}$ & $\mathbf{C T}$ & JK & GP & Monoptic $P$ & $\mathbf{C T}$ & JK & GP \\
\hline $3 \mathrm{~Hz}$ & 0.30 & 0.33 & 0.30 & $3 \mathrm{~Hz}$ & 0.19 & 0.22 & 0.22 & $3 \mathrm{~Hz}$ & 0 & 3 & 7 \\
\hline $6 \mathrm{~Hz}$ & 0.25 & 0.28 & 0.27 & $6 \mathrm{~Hz}$ & 0.21 & 0.28 & 0.21 & $6 \mathrm{~Hz}$ & 0 & 16 & 10 \\
\hline $12 \mathrm{~Hz}$ & 0.28 & 0.26 & 0.26 & $12 \mathrm{~Hz}$ & 0.13 & 0.13 & 0.12 & $12 \mathrm{~Hz}$ & -2 & 18 & 23 \\
\hline Dichoptic $R_{C}$ & CT & JK & GP & Dichoptic $R_{S}$ & CT & JK & GP & Dichoptic $P$ & CT & JK & GP \\
\hline $3 \mathrm{~Hz}$ & 0.38 & 0.46 & 0.45 & $3 \mathrm{~Hz}$ & 0.16 & 0.09 & 0.12 & $3 \mathrm{~Hz}$ & 6 & 33 & 11 \\
\hline $6 \mathrm{~Hz}$ & 0.41 & 0.49 & 0.46 & $6 \mathrm{~Hz}$ & 0.13 & 0.08 & 0.06 & $6 \mathrm{~Hz}$ & 16 & 0 & 26 \\
\hline $12 \mathrm{~Hz}$ & 0.42 & 0.46 & 0.43 & $12 \mathrm{~Hz}$ & 0.08 & 0.00 & 0.05 & $12 \mathrm{~Hz}$ & 13 & - & 18 \\
\hline $\begin{array}{c}\text { Monoptic- } \\
\text { Dichoptic } R_{C}\end{array}$ & CT & JK & GP & $\begin{array}{c}\text { Monoptic- } \\
\text { Dichoptic } R_{S}\end{array}$ & $\mathbf{C T}$ & JK & GP & $\begin{array}{l}\text { Monoptic- } \\
\text { Dichoptic } P\end{array}$ & CT & JK & GP \\
\hline $3 \mathrm{~Hz}$ & - & - & - & $3 \mathrm{~Hz}$ & 0.02 & 0.14 & 0.10 & $3 \mathrm{~Hz}$ & - & 27 & 4 \\
\hline $6 \mathrm{~Hz}$ & - & - & - & $6 \mathrm{~Hz}$ & 0.09 & 0.19 & 0.15 & $6 \mathrm{~Hz}$ & -31 & 25 & 4 \\
\hline $12 \mathrm{~Hz}$ & - & - & - & $12 \mathrm{~Hz}$ & 0.05 & 0.14 & 0.07 & $12 \mathrm{~Hz}$ & -17 & 22 & 30 \\
\hline
\end{tabular}


not shown. The $R_{C}$ values in the monoptic and dichoptic data were similar for all subjects (see also Table 1).

The estimates of $R_{S}$ (as a quantification of the lateral interactions) are also displayed as a function of temporal frequency (Figure 6, middle panels). For all subjects, the $R_{S}$ components were larger in the monoptic than in the dichoptic conditions. In addition, the estimated subcortical $R_{S}$ component was generally larger or of a same magnitude as the cortical (dichoptic) $R_{S}$ component. These data suggest that there is substantial subcortical contribution to the monoptic PFS modulation. In addition, the temporal frequency seems to have different influences on the cortical and subcortical $R_{S}$ components. While the magnitude of the cortical $R_{S}$ components decreases from 3 to $12 \mathrm{~Hz}$, the subcortical mechanisms seem to have a maximal $R_{S}$ component at about $6 \mathrm{~Hz}$. Further, there is considerable inter-individual variability in the $R_{S}$ values.

Figure 6 (bottom panels) shows the estimates of the relative phase $P$ for a minimal PFS displayed as a function of temporal frequency. As mentioned above, the $P$ values were disregarded when the difference between the maximal and minimal values of the PFS equivalent contrast was less than 3 times the average of the standard deviations at all data points. Generally, the phase at minimal PFS was positive, suggesting a phase lag of the mechanisms that respond to the surround stimulus (see Table 1).

\section{Discussion}

Our data are qualitatively similar to those of D'Antona et al.(2011). We both find that there are indications of cortical and subcortical lateral interaction mechanisms influencing the perception of flicker in a center field simultaneously modulated with a surround. Furthermore, we both find that the cortical component is relatively stronger at low temporal frequencies In preliminary experiments, Kozyrev et al. (2007) mentioned that they could see a lateral interaction when the test stimulus was presented monoptically but not when presented dichoptically, suggesting the presence of only subcortical mechanisms. Our data and those of D'Antona et al. (2011) are only partially in agreement with these anecdotic observations.

The suggestion of a subcortical and a cortical mechanism with different temporal dependencies is in qualitative agreement with other studies that show that temporal vision is determined by two channels: one with cortical origin at low temporal frequencies and a subcortical mechanism at higher temporal frequencies (Cass \& Alais, 2006).

As mentioned in the introduction, we used a matching stimulus that was presented to one eye whereas D'Antona et al. (2011) presented the matching stimulus to the two eyes. This might explain why our PFS values are larger than theirs, indicating that there is inter-ocular integration of contrast in the matching stimulus.
Another difference is that D'Antona et al. (2011) find that the subcortical component is generally smaller than the cortical component except at $12 \mathrm{~Hz}$. Furthermore, they find that the strength of the subcortical component does not depend on temporal frequency, whereas the strength of the cortical component decreases with temporal frequency. We confirm the low-pass characteristics of the cortical mechanism but find that the subcortical component is particularly strong at $6 \mathrm{~Hz}$, suggesting a band-pass characteristic.

To study whether the monoptic or dichoptic presentation of the matching stimulus indeed influenced the data, we performed a pilot study. In this pilot study, the center and surround test stimuli modulated at $6 \mathrm{~Hz}$ in-phase or in counterphase. Two subjects (CT and GP) participated. The same procedures as described above were adopted, but the matching stimuli were presented either monocularly and binocularly. Figure 7 (top and middle panels) shows the results of these measurements. It can be seen that a binocular matching task indeed led to a reduction of the equivalent contrast in the matching stimulus of about a factor of 2 .

We further estimated $R_{S}$ component with the monocular and binocular matching tasks. These results are shown in the lower panels of Figure 7. On the basis of these preliminary data, it is not possible to draw definite conclusions. However, the data of GP suggest that the subcortical component might indeed be relatively smaller in comparison with cortical component when the matching stimulus is presented binocularly. More data would be necessary to show whether there is indeed an influence of matching stimulus presentation. Other stimulus conditions also differ. Whereas D'Antona et al. (2011) use a surround with a $5^{\circ}$ outer diameter and a $27 \mathrm{~cd} / \mathrm{m}^{2}$ mean luminance, we used a $3^{\circ}$ surround and a mean luminance of $9 \mathrm{~cd} /$ $\mathrm{m}^{2}$. On the basis of previous data (Kremers et al., 2004; Teixeira \& Kremers, 2009) it is, however, unlikely that these difference influence the results.

In agreement with the data of D'Antona et al. (2011), we found that the strength of the cortical component decreases with increasing temporal frequencies. D'Antona et al. (2011) argue that object segmentation mechanisms may be an explanation for the cortical lateral interactions. An alternative explanation may be brightness induction (Ejima \& Takahashi, 1985), i.e. the change of the perceived intensity of a static region by the luminance of surrounding regions. This mechanism indeed has a low pass characteristic with a temporal cutoff frequency of about $2-5 \mathrm{~Hz}$ and small temporal phase lag (DeValois, Webster, DeValois, \& Lingelbach, 1986; Rossi \& Paradiso, 1996, 1999; Rossi, Rittenhouse, \& Paradiso, 1996; Blakeslee \& MacCourt, 2008). But, on the basis of our data, we find that it is not possible to precise which cortical mechanisms may be responsible for the dichoptic lateral interactions involved in the PFS in the center stimulus. 
CT

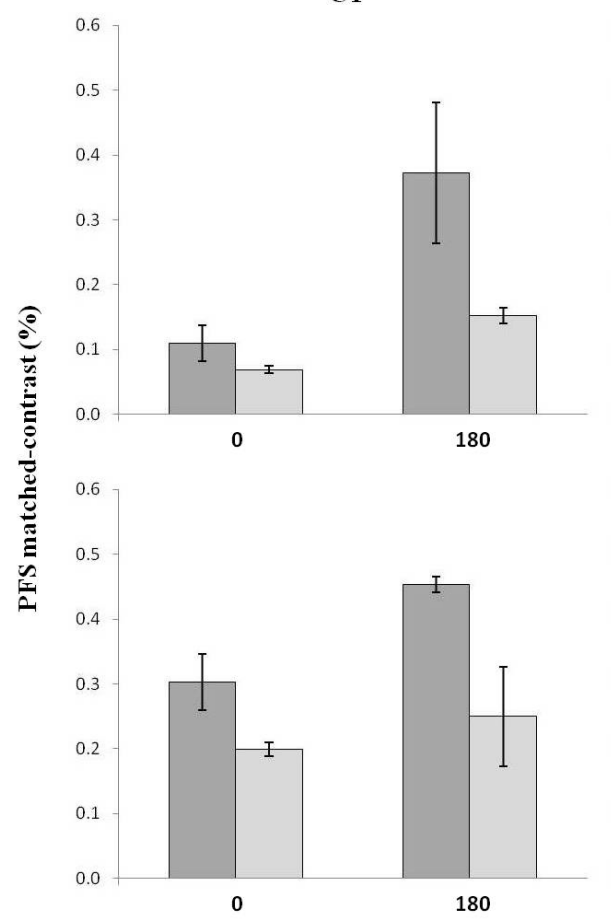

GP

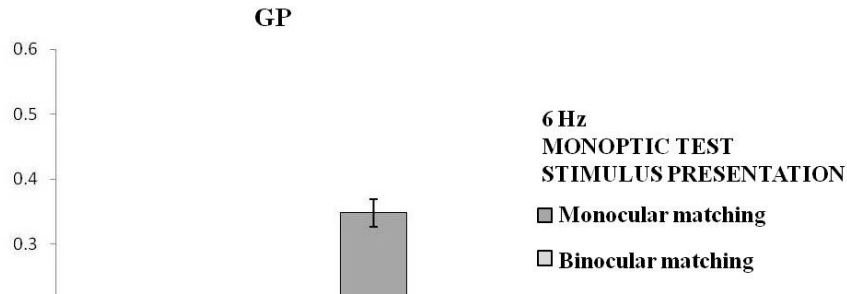

Phase-difference between center and surround stimuli (degrees)
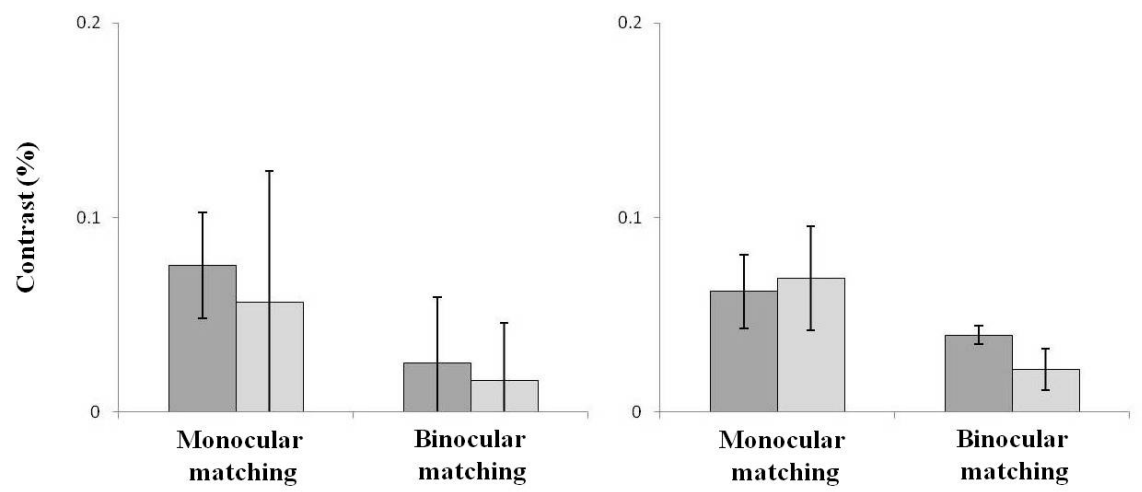

$6 \mathrm{~Hz}$

$\square$ Cortical $R s$ component

$\square$ Subcortical $R s$ component STIMULUS PRESENTATION

$\square$ Monocular matching

$\square$ Binocular matching

Figure 7. The PFS (mean \pm SD) in the center test stimulus plotted as a function of the phase difference between center and surround stimuli at $6 \mathrm{~Hz}$ for two subjects. The data are presented in monoptic (top) and dichoptic (middle) test conditions, and when the contrast in the matching stimulus was adjusted monocularly (dark bars) and binocularly (light bars). Bottom: The estimates of cortical and subcortical $R_{S}$ components when the contrast in the matching stimulus was adjusted monocularly (dark bars) and binocularly (light bars) In this case, $R_{S}=\left(\mathrm{PFS}_{180^{\circ}}-\mathrm{PFS}_{0^{\circ}}\right) / 2$, where $\mathrm{PFS}_{180^{\circ}}$ and $\mathrm{PFS}_{0^{\circ}}$ are the matched-contrasts in the matching stimulus when the center-surround test stimulus is modulated in counterphase and in-phase, respectively.

\section{Acknowledgement}

The authors would like to thank the support by German Research Council (DFG) grant KR 1317/9-1, CAPESDAAD PROBRAL \#182/2007 grant, CAPES-BMBF BRA09/010, and CNPq \#550671/2007-2. JK is Fellow in the Excellence Program of the Hertie Foundation.

\section{References}

Blakeslee, B., \& MacCourt, M.E. (2008). Nearly instantaneous brightness induction. Journal of Vision, 8(2), 1-8.

Cass, J., \& Alais, D. (2006). Evidence for two interacting temporal channels in human visual processing. Vision Research, 46, 2859-2868.

D'Antona, A.D., Kremers, J., \& Shevell, S.K. (2008). A cortical and a sub-cortical origin of lateral interactions in perceived temporal variation. Journal of Vision, 8 (6), abstract 358.

D'Antona, A.D., Kremers, J., \& Shevell, S.K. (2011). Contextual Influences on Perceived Temporal Variation Result from Two Separate Neural Mechanisms at Different Stages of the Visual System. Vision Research, (in submission).

DeValois, R.L., Webster, M.A., DeValois, K.K., \& Lingelbach, B. (1986). Temporal properties of brightness and color induction. Vision Research, 26, 887-897.

Ejima, Y., \& Takahashi, S. (1985). Apparent contrast of a sinusoidal grating in the simultaneous presence of peripheral gratings. Vision Research 25, 1223-1232.

Kilavik, B.E., Silveira, L.C., \& Kremers, J. (2003). Centre and surround responses of marmoset lateral geniculate neurons at different temporal frequencies. Journal of Physiology, 546(Pt 3), 903-19.

Kozyrev, V., Silveira, L.C.L., \& Kremers, J. (2007). Linking lateral interactions in flicker perception to lateral geniculate nucleus cell responses. Journal of Physiology, 581, 1083-1100.

Kremers, J., Kozyrev, V., Silveira, L.C., \& Kilavik, B.E. (2004). Lateral interactions in the perception of flicker and in the physiology of the 
lateral geniculate nucleus. Journal of Vision, 4, 643-63.

Kremers, J., \& Rimmele, U. (2007). The spatial extent of lateral interactions in flicker perception. Vision Research, 47(1), 16-21.

Rossi, A.F., \& Paradiso, M.A. (1996). Temporal limits of brightness induction and mechanisms of brightness perception. Vision Research, 36, 1391-1398.

Rossi, A.F., Rittenhouse, C.D., \& Paradiso, M.A. (1996). The representation of brightness in primary visual cortex. Science, 273,
1104-1107.

Rossi, A.F., \& Paradiso M.A. (1999). Neural correlates of perceived brightness in the retina, lateral geniculate nucleus, and striate cortex. Journal of Neuroscience, 19, 6145-6156.

Teixeira, C., \& Kremers, J. Cortical and subcortical origins of lateral interactions in flicker perception (2009). $20^{\text {th }}$ Symposium of the International Color Vision Society, Braga, Portugal. Abstract booklet, pp. 149. 